\title{
Interactions between cigarette smoking and polymorphisms of xenobiotic-metabolizing genes: The risk of oral leukoplakia
}

\author{
Yu-Fen Li ${ }^{\mathrm{a}}$, Fung-Chang Sung ${ }^{\mathrm{b}}$, Ming-Hsui Tsaic ${ }^{\mathrm{c}}$, Chun-Hung Hua ${ }^{\mathrm{c}}$, Chiu-Shong Liu ${ }^{\mathrm{d}}$, \\ Yao-Te Huang ${ }^{\mathrm{e}}$ and Chih-Ching Yeh ${ }^{\mathrm{b}, \mathrm{f}, *}$ \\ ${ }^{a}$ Graduate Institute of Biostatistics, College of Public Health, China Medical University, Taichung, Taiwan \\ ${ }^{\mathrm{b}}$ Department of Public Health, College of Public Health, China Medical University, Taichung, Taiwan \\ ${ }^{\mathrm{c}}$ Department of Otolaryngology, China Medical University Hospital, Taichung, Taiwan \\ ${ }^{\mathrm{d}}$ Department of Family Medicine, China Medical University Hospital, Taichung, Taiwan \\ ${ }^{\mathrm{e}}$ Department of Life Science, China Medical University, Taichung, Taiwan \\ ${ }_{\mathrm{f}}^{\mathrm{f}}$ School of Public Health, College of Public Health and Nutrition, Taipei Medical University, Taipei, Taiwan
}

\begin{abstract}
.
BACKGROUND: This case-control study investigates the role of xenobiotic-metabolizing genes, including glutathione Stransferases (GSTs) and cytochrome P450 1A1 (CYP1A1) and 2E1 (CYP2E1), in the susceptibility to oral potentially malignant disorders (OPMDs).

METHODS: The genotypes of GSTM1, GSTT1, GSTP1, CYP1A1*2C, and CYP2E1 PstI/Rsal polymorphisms were determined for 217 OPMD cases and 492 age- and sex-matched controls from a Taiwanese penitentiary.

RESULTS: Compared to the GSTM1-present genotype, the GSTM1-null genotype was significantly associated with increased risk of leukoplakia (odds ratio $[\mathrm{OR}]=1.46,95 \%$ confidence interval $[\mathrm{CI}]=1.01-2.10$ ). Similarly, compared to the CYP1A $* 2 \mathrm{C}$ $\mathrm{A} / \mathrm{G}+\mathrm{G} / \mathrm{G}$ genotype, the CYP1A1*2C A/A genotype was significantly associated with increased risk of leukoplakia $(\mathrm{OR}=1.64$, $95 \% \mathrm{CI}=1.12-2.40)$, particularly for smokers consuming $>13$ pack-years of cigarettes $(\mathrm{OR}=2.40,95 \% \mathrm{CI}=1.40-4.11)$ (Interaction $P=0.039)$. In addition, participants with 4-5 risk genotypes (OR $>1)$ experienced higher risks for leukoplakia than those with $0-1$ risk genotypes $(\mathrm{OR}=3.19,95 \% \mathrm{CI}=1.65-6.15)$ (Trend test $P=0.001$ ).

CONCLUSIONS: Our findings suggest that the CYP1A1*2C A/A genotype may increase the risk of leukoplakia, especially for heavy smokers. Xenobiotic-metabolizing genes may simultaneously modulate this disease risk. These observations require further confirmation with larger samples.
\end{abstract}

Keywords: Glutathione S-transferases, cytochrome P450 1A1, cytochrome P450 2E1, polymorphisms, oral potentially malignant disorders, cigarette smoking

\section{Introduction}

Oral cancer is one of most commonly diagnosed cancers, with an estimated 263,900 new cases and 128,000 deaths from this disease occurring in 2008

${ }^{*}$ Corresponding author: Chih-Ching Yeh, School of Public Health, College of Public Health and Nutrition, Taipei Medical University, $250 \mathrm{Wu}-H$ sing Street, Taipei City 110, Taiwan. Tel.: +886227361661 ext. 6534; Fax: +886 22738 4831; E-mail: ccyeh@tmu.edu.tw. worldwide. The age-standardized incidence was 5.2 per 100,000 for men [1]. In Taiwan, oral cancer is the fourth leading cause of cancer death in men and is the most common cancer in people aged 24-45 years [2]. The age-standardized mortality rate for oral cancer in Taiwan was 15.2 per 100,000 people in 2011 , which is substantially higher than in other countries. The median age at death from the disease in Taiwan in 2011 is 56 years in men, which is the youngest age at death among all cancer types in men. 
Oral potentially malignant disorders (OPMDs) are associated with oral malignant transformation [3-5], and researchers have not made significant improvements in the early diagnosis of oral cancer in the last few decades [6]. Many studies have shown that cigarette smoking, excess alcohol consumption, and betel (areca) quid chewing are important risk factors for oral cancer and OPMDs [7-10]. Studies in Taiwan have demonstrated the synergistic effects of these habits on the risk of oral cancer and OPMDs [7,11].

Molecular epidemiological studies have now provided evidence that a person's susceptibility to cancer is mediated by genetic and environmental factors [12]. Most carcinogenic chemicals require the metabolic activation of phase-I enzymes (e.g., cytochrome P450; CYP) or detoxification by phase-II enzymes (e.g., glutathione S-transferases; GSTs) [13]. CYP1A1 polymorphisms increase microsomal catalytic activity for converting procarcinogens, including polycyclic aromatic hydrocarbons (PAHs) and aromatic amines [14]. CYP2E1 activates tobaccospecific nitrosamines and modulates the formation of safrole-DNA adducts among areca quid chewers [15, 16]. GSTM1 and GSTT1 detoxify hydrophobic electrophiles derived from the metabolism of xenobiotics, including PAHs [17]. Because most carcinogens present in tobacco, alcohol, and betel nut require some form of biotransformation to exert their biological effects, the genetic polymorphisms of the enzymes involved in biotransformation pathways may modulate cancer risks.

Several studies, 3 meta-analyses, have suggested that GSTM1 and CYP1A1 polymorphisms are related to the risk of oral squamous cell carcinoma [18-20]. However, studies on the association between polymorphisms of xenobiotic-metabolizing genes and OPMD risk are controversial. Therefore, the goal of this casecontrol study assesses the influences of genetic polymorphisms of the GSTM1, GSTT1, GSTP1, CYP1A1, and CYP2E1 genes and their interactions with environmental factors such as smoking in conferring OPMD susceptibility in a male prison population in Taiwan.

\section{Materials and methods}

\subsection{Ethnic statement}

All prisoners were invited to join the study after hearing a detailed description of the research purposes. After giving written informed consent for oral health screening, each participant received an oral examination and completed a structured questionnaire. Confirmed OPMD cases and health controls were subsequently invited to join a genetic study. Those participants also signed informed consent for the genetic study and provided blood specimens. Any potential participants who declined to participate or otherwise did not participate were nonetheless eligible for oral health screening, and were not disadvantaged in any way by not participating in the study. The Ethics Review Committee of China Medical University and the Institutional Review Board at China Medical University Hospital approved the study protocol.

\subsection{Study subjects}

We conducted oral screening for OPMDs in all prisoners in the largest male penitentiary in Taiwan from October 2007 to March 2008. Among an estimated average of 4000 inmates, 3059 men $(76.48 \%)$ participated in the screening and completed the questionnaire. Two experienced otolaryngologists conducted the oral examinations and evaluated the inmates based on World Health Organization (WHO) criteria [21]. Cases of OPMD were diagnosed as with leukoplakia, erythroplakia, or oral submucous fibrosis. Prisoners with normal screening results served as healthy controls. After excluding 14 inmates with oral cancer and 30 inmates with other minor abnormal oral conditionlike ulcers, the potential participants for the genetic study included 488 cases and 2527 controls. We invited all cases to participate, and randomly selected 3 times that number of age- (5-year age groups) and sexmatched controls to participate in the genetic study. All participants were asked to provide $10 \mathrm{~mL}$ of venous blood.

\subsection{Evaluation of risk factors}

Each participant completed a self-administered questionnaire to provide information on sociodemographic characteristics, lifestyle factors (including cigarette smoking, alcohol consumption, and betel nut chewing), environmental tobacco smoke, height, weight, and personal and family medical histories. The family cancer history was extended only to first-degree relatives. The body mass index (BMI) was calculated as weight $/$ height $^{2}\left(\mathrm{~kg} / \mathrm{m}^{2}\right)$. Participants who reported cigarette smoking, alcohol consumption, and betel nut chewing were classified into 3 groups: current users (who had used for more than $1 \mathrm{y}$ ), nonusers, and exusers (who had stopped use for at least $6 \mathrm{mo}$ ). Total cigarette consumption in pack-years was estimated using daily consumption multiplied by years of use. 


\subsection{Genotyping}

Genomic DNA was extracted from buffy coat cells using a DNA extraction kit (Qiagen, Chatsworth CA). The extracted DNA was dissolved in a Tris-EDTA buffer ( $\mathrm{pH} \mathrm{8.0)}$ ) to a final concentration of $50 \mathrm{ng} / \mu \mathrm{L}$. Polymorphisms were identified by polymerase chain reaction (PCR) assays for GSTM1, GSTT1, and CYP1 $\mathrm{A} 1 * 2 \mathrm{C}(\mathrm{A} 4889 \mathrm{G})$ (rs1048943) or combined with restriction fragment-length polymorphism (RFLP) assays for GSTP1 Ile105Val (rs1695), CYP2E1 PstI (-1293G>C) (rs3813867), and CYP2E1 RsaI (-1053C> T) (rs2031920). All PCRs were performed in a $25-\mu \mathrm{L}$ final volume containing $5 \mathrm{pM}$ of each primer, $50 \mathrm{ng}$ genomic DNA, $1.5 \mathrm{mM} \mathrm{MgCl} 2,200 \mu \mathrm{M}$ dNTPs, and 1.0 unit of Taq DNA polymerase in the buffer provided by the manufacturer. Amplification was performed using a Mastercycler gradient thermocycler (Eppendorf, Hamburg, Germany) for the PCR.

The GSTM1 and GSTT1 genes were detected using a multiple PCR assay with the following primers: GSTM1 (5'-TGC CCT ACT TGA TTG ATG GG-3' and 5'-CTG GAT TGT AGC AGA TCA TGC-3'), GSTT1 (5'-TTC CTT ACT GGT CCT CAC ATC TC3' and 5'-CAC CGG ATC ATG GCC AGC A-3'), and $\beta$-globin as an internal control (5'-CAC AAC TGT GTT CAC TAG C-3' and 5'-CAA CTT CAT CCA CGT TCA CC-3'). People without intact GSTT1 or GSTM1 genes showed no amplification of the 480bp GSTT1 fragment or the 273-bp GSTM1 fragment and a positive internal control [22]. The CYP1A $1 * 2 \mathrm{C}$ (A4889G) polymorphism was identified using one sense primer (5'-GAA CTG CCA CTT CAG CTG TCT-3') and 2 antisense primers, differing in the last base, for the A allele (5'-AAG ACC TCC CAG CGG GCA AT-3') and G allele (5'-AAG ACC TCC CAG CGG GCA AC-3'). Each sample was tested in 2 parallel reactions, with the same sense primer and one antisense primer. Amplification occurred in both tubes (heterozygotes) or only the tubes containing the exact matching antisense primer (homozygotes) [23]. The 176-bp PCR product of the GSTP1 A/G (Ile105Val) polymorphism was amplified with the primers 5'CCT CTC CCT TTC CTC TGT TC-3' (sense) and 5'-CAG GTG AGG GGG ACA TCT-3' (antisense) and digested with Alw26I (New England BioLabs, Beverly, MA). The Val allele showed 91- and 85-bp fragments following digestion and polyacrylamide gel electrophoresis, but Alw26I did not digest the Ile allele [22]. The PstI and RsaI polymorphic sites of the CYP2E1 gene were determined using the following primers: sense, 5'-CCA GTC GAG TCT ACA TTG TCA-3' and antisense, 5' -TTC ATT CTG TCT TCT AAC TGG-3'. The 410-bp PCR product was digested with PstI and RsaI (Fermentas, Glen Burnie, MD). The presence of the PstI restriction site produced 2 fragments of 120 and 290 bp for the $\mathrm{C}$ allele (the $\mathrm{G}$ allele was not digested). However, the $\mathrm{C}$ allele was cut into 360- and 50-bp fragments indicating the presence of the RsaI restriction site (the $\mathrm{T}$ allele was not digested) [24]. A random 10\% sample retested for quality control showed $100 \%$ concordance for all polymorphisms, and all laboratory personnel were blinded to the oral premalignant status of the samples.

\subsection{Statistical analysis}

The differences between cases and controls were compared using either the Student's $t$ test for continuous variables or a chi-square test for categorical data. Observed genotype frequencies for all the polymorphisms in the controls were examined to determine their deviation from the Hardy-Weinberg equilibrium (HWE) using a goodness-of-fit $\chi^{2}$ test with one degree of freedom. Conditional logistic regression was used to calculate the odd ratios (ORs) and 95\% confidence intervals (CIs) for the risk of OPMD associated with genetic polymorphisms. The multivariate logistic regression models included covariates such as BMI, cigarette smoking, alcohol consumption, and betel nut chewing to estimate adjusted ORs and 95\% CIs. Multiplicative interaction analysis with a likelihood ratio test was conducted to evaluate the genesmoking interactions on OPMD risk. Because more than $95 \%$ of the study participants smoked habitually, we used the median pack-years of cigarettes (13 pack-years) among the controls to evaluate the genesmoking interaction. The combined effects of the highrisk genotypes (genotypes with ORs of $>1$, i.e., null for GSTM1 and GSTT1, and A/G+G/G for GSTP1, A/A for CYP1A1, and $\mathrm{G} / \mathrm{C}+\mathrm{C} / \mathrm{C}$ for CYP2E1 PstI) were estimated using indicator variables to denote various combinations of joint exposures. All analyses were performed using the SAS statistical package (ver. 9.1 for Windows; SAS Institute, Cary, NC), and a twosided $P<0.05$ was served as the criterion for statistical significance.

\section{Results}

Among the 3015 potential participants (488 cases and 2527 controls), 716 inmates agreed to participate 
Table 1

Comparisons of characteristics between cases with oral potentially malignant disorders and healthy controls

\begin{tabular}{|c|c|c|c|}
\hline Variable $^{\mathrm{a}}$ & Cases $N=217(\%)$ & Controls $N=492(\%)$ & $P$ value $^{\mathrm{c}}$ \\
\hline Mean age (SD), years & $38.8(9.2)$ & $38.2(9.1)$ & 0.46 \\
\hline BMI (SD), $\mathrm{kg} / \mathrm{m}^{2}$ & & & 0.003 \\
\hline$<18.5$ & $3(1.4)$ & $7(1.4)$ & \\
\hline $18.5-24$ & $96(44.2)$ & $292(59.4)$ & \\
\hline $24-27$ & $81(37.3)$ & $130(26.4)$ & \\
\hline$\geqslant 27$ & $37(17.1)$ & $63(12.8)$ & \\
\hline Educational level & & & 0.05 \\
\hline Below elementary school & $43(20.2)$ & $83(16.9)$ & \\
\hline Junior high school & $108(50.7)$ & $218(44.4)$ & \\
\hline Above senior high school & $62(29.1)$ & $190(38.7)$ & \\
\hline Alcohol consumption & & & 0.77 \\
\hline None & $72(33.2)$ & $177(36.0)$ & \\
\hline Current drinker & $80(36.9)$ & $173(35.2)$ & \\
\hline Ex-drinker & $65(30.0)$ & $142(28.8)$ & \\
\hline Betel nut chewing & & & 0.023 \\
\hline None & $53(24.4)$ & $171(34.8)$ & \\
\hline Current chewer & $65(30.0)$ & $130(26.5)$ & \\
\hline Ex-chewer & 99 (45.6) & $190(38.7)$ & \\
\hline Cigarette smoking & & & 0.12 \\
\hline None & $5(2.3)$ & $24(4.9)$ & \\
\hline Current smoker & $201(92.6)$ & $431(87.6)$ & \\
\hline Ex-smoker & $11(5.1)$ & $37(7.5)$ & \\
\hline Pack-years of cigarettes ${ }^{b}$ & & & 0.26 \\
\hline$\leqslant 7.5$ & $49(22.6)$ & $143(29.1)$ & \\
\hline $7.6-13.0$ & $51(23.5)$ & $117(23.8)$ & \\
\hline $13.1-20.0$ & $59(27.2)$ & $124(25.2)$ & \\
\hline$>20.1$ & $58(26.7)$ & $108(22.0)$ & \\
\hline Family cancer history & & & 0.65 \\
\hline No & $165(76.0)$ & $381(77.4)$ & \\
\hline Yes & $52(24.0)$ & $111(22.6)$ & \\
\hline
\end{tabular}

a Numbers not being equal to the total number was due to missing data.

${ }^{\mathrm{b}}$ Quartile pack-years of cigarettes among the controls.

${ }^{\mathrm{c}}$ Student's $t$-test or Chi-square test.

in the genetic study (223 cases and 493 controls). Because 7 specimens failed in genotyping, 217 cases and 492 controls were used for the statistical analysis. Among the OPMD cases, 170 participants had leukoplakia (78.3\%), 78 had submucous fibrosis (35.9\%), and 7 had erythroplakia (3.2\%). Table 1 shows the sociodemographic characteristics and lifestyle factors of the study participants. The age of participants ranged from 20-76 years for cases and 20-70 years for controls, with an overall average of 38 years. Compared to the controls, the study cases were more obese, chewed betel nut, and were less educated $(P<0.05)$. The multivariate logistic regression models included these factors to adjust for their potentially confounding effects.

Table 2 shows the genotypic distributions of xenobiotic-metabolizing genes for cases and controls. The genotype distributions were in agreement with HWE, except for the GSTP1 polymorphism $(P=0.042)$. However, the frequencies of the variant alleles of the polymorphisms in our study are compared with previously published reports in Taiwan. Because of the rel- atively low frequencies of variant alleles for the genes in this study, any genotype featuring one or more variant alleles was combined for further analyses. The OPMD risk marginally increased for people featuring the GSTM1-null genotype $(\mathrm{OR}=1.40 ; 95 \% \mathrm{CI}=$ 1.01-1.96) and the CYP1A1*2C A/A genotype (OR = $1.39 ; 95 \% \mathrm{CI}=0.99-1.95)$. These increased genetic effects were pronounced in cases with leukoplakia with corresponding ORs of 1.46 (95\% CI $=1.01-2.10)$ and 1.64 (95\% CI $=1.12-2.40)$, respectively. There was no significant association between other polymorphisms and the risk of OPMD or specific disorders. We selected CYP2E1 PstI for further analyses because CYP2E1 PstI and RsaI were closely linked (D' $=0.94$, $P<0.001)$.

Table 3 shows the interactions between the polymorphisms of xenobiotic-metabolizing genes and cigarette smoking and their effects on OPMD risk. Interactions between CYP1A $1 * 2 \mathrm{C}$ polymorphisms and smoking were slightly significant for OPMD and especially significant for leukoplakia (Interaction $P=0.039$ ). 
Table 2

Associations between xenobiotic-metabolizing polymorphisms and the risk of oral potentially malignant disorders

\begin{tabular}{|c|c|c|c|c|c|c|c|}
\hline \multirow[b]{2}{*}{ Genotype } & \multirow{2}{*}{$\begin{array}{c}\text { Controls } \\
N=492(\%)\end{array}$} & \multicolumn{2}{|c|}{ Cases } & \multicolumn{2}{|c|}{ Leukoplakia } & \multicolumn{2}{|c|}{ Submucous fibrosis } \\
\hline & & $N=217(\%)$ & OR $(95 \% \mathrm{CI})^{\mathrm{a}}$ & $N=170(\%)$ & OR $(95 \% \mathrm{CI})^{\mathrm{a}}$ & $N=78(\%)$ & OR $(95 \% \mathrm{CI})^{\mathrm{a}}$ \\
\hline \multicolumn{8}{|l|}{ GSTM1 } \\
\hline Present & 249 (50.6) & $92(42.4)$ & 1.00 & $72(42.4)$ & 1.00 & $35(44.9)$ & 1.00 \\
\hline Null & $243(49.4)$ & 125 (57.6) & $1.40(1.01-1.96)^{*}$ & $98(57.7)$ & $1.46(1.01-2.10)^{*}$ & $43(55.1)$ & $1.27(0.77-2.10)$ \\
\hline \multicolumn{8}{|l|}{ GSTT1 } \\
\hline Present & $191(38.8)$ & $84(38.7)$ & 1.00 & $64(37.7)$ & 1.00 & $33(42.3)$ & 1.00 \\
\hline Null & $301(61.2)$ & $133(61.3)$ & $1.05(0.75-1.47)$ & $106(62.4)$ & $1.08(0.74-1.57)$ & $45(57.7)$ & $0.89(0.54-1.47)$ \\
\hline \multicolumn{8}{|l|}{ GSTP1 } \\
\hline $\mathrm{A} / \mathrm{A}$ & $336(68.3)$ & $146(67.3)$ & 1.00 & $113(66.5)$ & 1.00 & $51(65.4)$ & 1.00 \\
\hline $\mathrm{A} / \mathrm{G}$ & $133(27.0)$ & $57(26.3)$ & $1.01(0.69-1.48)$ & $45(26.5)$ & $1.05(0.69-1.59)$ & $23(29.5)$ & $1.18(0.67-2.07)$ \\
\hline $\mathrm{G} / \mathrm{G}$ & $23(4.7)$ & $14(6.5)$ & $1.60(0.78-3.25)$ & $12(7.1)$ & $1.85(0.87-3.92)$ & $4(5.1)$ & $1.32(0.42-4.17)$ \\
\hline $\mathrm{A} / \mathrm{G}+\mathrm{G} / \mathrm{G}$ & $156(31.7)$ & $71(32.7)$ & $1.10(0.77-1.56)$ & $57(33.5)$ & $1.16(0.79-1.70)$ & $27(34.6)$ & $1.20(0.71-2.04)$ \\
\hline \multicolumn{8}{|l|}{ CYP1A1*2C } \\
\hline $\mathrm{A} / \mathrm{A}$ & $280(56.9)$ & $141(65.0)$ & 1.00 & $117(68.8)$ & 1.00 & $47(60.3)$ & 1.00 \\
\hline $\mathrm{A} / \mathrm{G}$ & $172(35.0)$ & $58(26.7)$ & $0.67(0.46-0.97)^{*}$ & $41(24.1)$ & $0.57(0.38-0.86)^{*}$ & $24(30.8)$ & $0.83(0.48-1.44)$ \\
\hline $\mathrm{G} / \mathrm{G}$ & $40(8.1)$ & $18(8.3)$ & $0.95(0.52-1.75)$ & $12(7.1)$ & $0.81(0.40-1.64)$ & $7(9.0)$ & $1.10(0.45-2.67)$ \\
\hline $\mathrm{A} / \mathrm{G}+\mathrm{G} / \mathrm{G}$ & $212(43.1)$ & $76(35.0)$ & $0.72(0.51-1.01)$ & $53(31.2)$ & $0.61(0.42-0.90)^{*}$ & $31(39.7)$ & $0.88(0.53-1.46)$ \\
\hline $\mathrm{A} / \mathrm{G}+\mathrm{G} / \mathrm{G}$ & $212(43.1)$ & $76(35.0)$ & 1.00 & $53(31.2)$ & 1.00 & $31(39.7)$ & 1.00 \\
\hline $\mathrm{A} / \mathrm{A}$ & $280(56.9)$ & $141(65.0)$ & $1.39(0.99-1.95)$ & $117(68.8)$ & $1.64(1.12-2.40)^{*}$ & $47(60.3)$ & $1.14(0.68-1.89)$ \\
\hline \multicolumn{8}{|c|}{ CYP2E1 PstI $(-1293 G>C)$} \\
\hline $\mathrm{G} / \mathrm{G}$ & $306(62.2)$ & $123(56.7)$ & 1.00 & $91(53.5)$ & 1.00 & $51(65.4)$ & 1.00 \\
\hline $\mathrm{G} / \mathrm{C}$ & $164(33.3)$ & $83(38.3)$ & $1.27(0.90-1.80)$ & $69(40.6)$ & $1.43(0.97-2.09)$ & $23(29.5)$ & $0.86(0.49-1.48)$ \\
\hline $\mathrm{C} / \mathrm{C}$ & $22(4.5)$ & $11(5.1)$ & $1.22(0.57-2.61)$ & $10(5.9)$ & $1.47(0.66-3.26)$ & $4(5.1)$ & $1.07(0.34-3.35)$ \\
\hline $\mathrm{G} / \mathrm{C}+\mathrm{C} / \mathrm{C}$ & $186(37.8)$ & $94(43.4)$ & $1.27(0.91-1.77)$ & $79(46.5)$ & $1.43(0.99-2.06)$ & $27(34.6)$ & $0.88(0.52-1.49)$ \\
\hline \multicolumn{8}{|c|}{ CYP2E1 RsaI $(-1053 \mathrm{C}>\mathrm{T})$} \\
\hline $\mathrm{T} / \mathrm{T}$ & 303 (61.6) & $121(55.8)$ & 1.00 & $91(53.5)$ & 1.00 & $49(62.8)$ & 1.00 \\
\hline $\mathrm{C} / \mathrm{T}$ & $162(32.9)$ & $85(39.2)$ & $1.36(0.96-1.92)$ & $70(41.2)$ & $1.49(1.02-2.18)^{*}$ & $24(30.8)$ & $0.95(0.55-1.64)$ \\
\hline $\mathrm{C} / \mathrm{C}$ & $27(5.5)$ & $11(5.1)$ & $0.96(0.45-2.03)$ & $9(5.3)$ & $1.01(0.45-2.18)$ & $5(6.4)$ & $1.03(0.37-2.93)$ \\
\hline $\mathrm{C} / \mathrm{T}+\mathrm{C} / \mathrm{C}$ & $189(38.4)$ & $96(44.2)$ & $1.30(0.93-1.81)$ & $79(46.5)$ & $1.42(0.98-2.04)$ & $29(37.2)$ & $0.96(0.58-1.61)$ \\
\hline
\end{tabular}

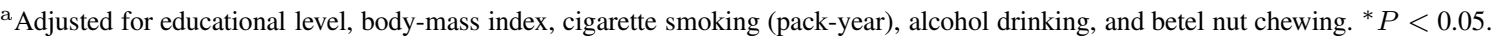

Among prisoners who had smoked $>13$ pack-years, the CYP1A1*2C A/A genotype was significantly associated with a 2.40-fold greater risk of leukoplakia compared to the CYP1A1*2C A/G+G/G genotype (OR $=2.40,95 \% \mathrm{CI}=1.40-4.11)$. Although other polymorphisms did not significantly interact with smoking in our study, the elevated leukoplakia risks of the GSTM1-null and CYP2E1*PstI G/C+C/C genotypes were substantially higher in prisoners who had smoked $>13$ pack-years than in those who had smoked $\leqslant$ 13 pack-years. Among heavy smokers, ORs for the GSTM1-null and CYP2E1*PstI G/C+C/C genotypes were $1.64(95 \% \mathrm{CI}=0.99-2.73)$ and $1.65(95 \% \mathrm{CI}=$ 1.01-2.69), respectively.

As Table 4 shows, participants exhibiting more risk genotypes (genotypes with an OR $>1$ ) faced a greater OPMD risk (Trend test $P=0.01$ ). Participants who demonstrated 4 or 5 of the putative risk genotypes showed a significantly higher risk of OPMD (OR = $2.15 ; 95 \% \mathrm{CI}=1.22-3.77)$ compared to those who featured none or one putative risk genotype. These combined genetic effects were further extended for leukoplakia (Trend test $P=0.001$ ) with a comparable OR of $3.19(95 \% \mathrm{CI}=1.65-6.15)$.

\section{Discussion}

This is the first case-control study with a relatively large sample to simultaneously investigate the association among 5 polymorphisms of xenobioticmetabolizing genes and OPMD risk in a male ethnic Chinese (i.e., Taiwanese) population in Taiwan. Results showed that the GSTM1-null and CYP1A1*2C A/A genotypes were positively associated with a risk of OPMDs, particularly leukoplakia. This increased risk was further elevated for heavy cigarette smokers. In addition, the gene-dosage effects of the 5 genes were found for OPMD and leukoplakia. These findings suggest that the genes involved in biotransformation may simultaneously participate in OPMD development.

The allelic frequencies of the GSTM1-null genotype and CYP1A $1 * 2 \mathrm{C} \mathrm{G}$ allele among healthy controls in this study $(49.4 \%$ and $25.6 \%$, respectively) were in agreement with previous reports in Taiwan [25]. The frequency of the GSTM1-null genotype in the control group ranged from $24 \%$ to $58.9 \%$, with considerable variation depending on the study area. Frequencies were lower in India (24\%-37\%), moderate in Japan 
Table 3

Associations between genotypes and risk of oral potentially malignant disorders stratified by cigarette use

\begin{tabular}{|c|c|c|c|c|c|c|c|c|}
\hline \multirow[b]{2}{*}{ Genotype } & \multirow[b]{2}{*}{$\begin{array}{c}\text { Pack-years of } \\
\text { cigarette }\end{array}$} & \multirow{2}{*}{$\begin{array}{c}\text { Controls } \\
N=492\end{array}$} & \multicolumn{2}{|r|}{ All cases } & \multicolumn{2}{|c|}{ Leukoplakia } & \multicolumn{2}{|c|}{ Submucous fibrosis } \\
\hline & & & $N=217$ & OR $(95 \% \mathrm{CI})^{\mathrm{a}}$ & $N=170$ & OR $(95 \% \mathrm{CI})^{\mathrm{a}}$ & $N=78$ & OR $(95 \% \mathrm{CI})^{\mathrm{a}}$ \\
\hline \multicolumn{9}{|l|}{ GSTM1 } \\
\hline Present & $\leqslant 13$ & 126 & 45 & 1.00 & 34 & 1.00 & 21 & 1.00 \\
\hline Null & $\leqslant 13$ & 134 & 55 & $1.17(0.70-1.79)$ & 40 & $1.13(0.65-1.96)$ & 21 & $1.06(0.53-2.13)$ \\
\hline Present & $>13$ & 123 & 47 & 1.00 & 38 & 1.00 & 14 & 1.00 \\
\hline Null & $>13$ & 109 & 70 & $1.58(0.98-2.52)$ & 58 & $1.64(0.99-2.73)$ & 22 & $1.43(0.67-3.07)$ \\
\hline$P$ for interaction & & & & 0.37 & & 0.29 & & 0.58 \\
\hline \multicolumn{9}{|l|}{ GSTT1 } \\
\hline Present & $\leqslant 13$ & 97 & 38 & 1.00 & 30 & 1.00 & 13 & 1.00 \\
\hline Null & $\leqslant 13$ & 163 & 62 & $1.15(0.70-1.90)$ & 44 & $1.05(0.60-1.84)$ & 29 & $1.52(0.72-3.18)$ \\
\hline Present & $>13$ & 94 & 46 & 1.00 & 34 & 1.00 & 20 & 1.00 \\
\hline Null & $>13$ & 138 & 71 & $0.99(0.62-1.60)$ & 62 & $1.15(0.68-1.92)$ & 16 & $0.54(0.25-1.17)$ \\
\hline$P$ for interaction & & & & 0.71 & & 0.69 & & 0.03 \\
\hline \multicolumn{9}{|l|}{ GSTP1 } \\
\hline $\mathrm{A} / \mathrm{A}$ & $\leqslant 13$ & 176 & 60 & 1.00 & 43 & 1.00 & 24 & 1.00 \\
\hline $\mathrm{A} / \mathrm{G}+\mathrm{G} / \mathrm{G}$ & $\leqslant 13$ & 84 & 40 & $1.38(0.84-2.27)$ & 31 & $1.54(0.88-2.69)$ & 18 & $1.68(0.82-3.42)$ \\
\hline $\mathrm{A} / \mathrm{A}$ & $>13$ & 160 & 86 & 1.00 & 70 & 1.00 & 27 & 1.00 \\
\hline $\mathrm{A} / \mathrm{G}+\mathrm{G} / \mathrm{G}$ & $>13$ & 72 & 31 & $0.84(0.50-1.40)$ & 26 & $0.88(0.51-1.51)$ & 9 & $0.73(0.31-1.71)$ \\
\hline$P$ for interaction & & & & 0.15 & & 0.14 & & 0.18 \\
\hline \multicolumn{9}{|l|}{ CYP1A1*2C } \\
\hline $\mathrm{A} / \mathrm{G}+\mathrm{G} / \mathrm{G}$ & $\leqslant 13$ & 154 & 60 & 1.00 & 45 & 1.00 & 26 & 1.00 \\
\hline $\mathrm{A} / \mathrm{A}$ & $\leqslant 13$ & 106 & 40 & $1.08(0.66-1.75)$ & 29 & $1.15(0.66-2.01)$ & 16 & $1.16(0.57-2.34)$ \\
\hline $\mathrm{A} / \mathrm{G}+\mathrm{G} / \mathrm{G}$ & $>13$ & 126 & 81 & 1.00 & 72 & 1.00 & 21 & 1.00 \\
\hline $\mathrm{A} / \mathrm{A}$ & $>13$ & 106 & 36 & $1.83(1.13-2.95)^{*}$ & 24 & $2.40(1.40-4.11)^{*}$ & 15 & $1.11(0.52-2.36)$ \\
\hline$P$ for interaction & & & & 0.09 & & 0.039 & & 0.97 \\
\hline \multicolumn{9}{|l|}{ CYP2E1*PstI } \\
\hline $\mathrm{G} / \mathrm{G}$ & $\leqslant 13$ & 167 & 60 & 1.00 & 46 & 1.00 & 26 & 1.00 \\
\hline $\mathrm{G} / \mathrm{C}+\mathrm{C} / \mathrm{C}$ & $\leqslant 13$ & 93 & 40 & $1.19(0.72-1.96)$ & 28 & $1.12(0.64-1.99)$ & 16 & $1.16(0.56-2.38)$ \\
\hline $\mathrm{G} / \mathrm{G}$ & $>13$ & 139 & 63 & 1.00 & 45 & 1.00 & 25 & 1.00 \\
\hline $\mathrm{G} / \mathrm{C}+\mathrm{C} / \mathrm{C}$ & $>13$ & 93 & 54 & $1.29(0.81-2.04)$ & 51 & $1.65(1.01-2.69)^{*}$ & 11 & $0.64(0.29-1.42)$ \\
\hline$P$ for interaction & & & & 0.93 & & 0.43 & & 0.26 \\
\hline
\end{tabular}

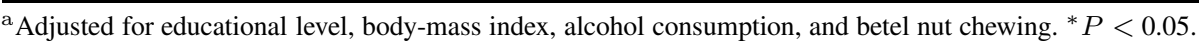

Table 4

Number of risk-genotypes of GSTM1, GSTT1, GSTP1, CYP1A1, and CYP2E1 and oral potentially malignant disorders

\begin{tabular}{|c|c|c|c|c|c|c|c|}
\hline \multirow[b]{2}{*}{ Number of risk genotypes ${ }^{\mathrm{a}}$} & \multirow{2}{*}{$\begin{array}{c}\text { Controls } \\
N=492\end{array}$} & \multicolumn{2}{|c|}{ All cases } & \multicolumn{2}{|c|}{ Leukoplakia } & \multicolumn{2}{|c|}{ Submucous fibrosis } \\
\hline & & $N=217$ & OR $(95 \% \mathrm{CI})^{\mathrm{b}}$ & $N=170$ & OR $(95 \% \mathrm{CI})^{\mathrm{b}}$ & $N=78$ & OR $(95 \% \mathrm{CI})^{\mathrm{b}}$ \\
\hline $0-1$ & 102 & 33 & 1.00 & 20 & 1.00 & 20 & 1.00 \\
\hline 2 & 164 & 69 & $1.41(0.86-2.33)$ & 55 & $1.95(1.07-3.56)$ & 20 & $0.69(0.34-1.39)$ \\
\hline 3 & 156 & 70 & $1.52(0.92-2.51)$ & 57 & $2.06(1.13-3.75)$ & 23 & $0.84(0.42-1.69)$ \\
\hline $4-5$ & 70 & 45 & $2.15(1.22-3.77)$ & 38 & $3.19(1.65-6.15)$ & 15 & $1.14(0.52-2.48)$ \\
\hline$P$ for trend & & & 0.01 & & 0.001 & & 0.68 \\
\hline
\end{tabular}

${ }^{\mathrm{a}}$ The risk genotype was defined as the genotype with an OR of $>1$ as shown in Table 2. ${ }^{\mathrm{b}}$ Adjusted for educational level, body-mass index, cigarette smoking (pack-year), alcohol consumption, and betel nut chewing. ${ }^{*} P<0.05$.

$(39.8 \%-48.7 \%)$ and South America $(38.2 \%-48.7 \%)$, and higher in Europe and the United States $(51 \%-$ $54.8 \%$ ) [18]. The CYP1A1*2C polymorphism exhibited large differences between countries. The $\mathrm{G}$ allele frequency in our controls was higher than in populations in India (12\%) [26], but substantially lower than those reported for a Brazilian population (42\%) [27].

CYP1A1 plays a well-established role in the metabolism of tobacco [28]. Buccal cells of the oral cavity exhibit a tissue-specific expression of this isoform [29].
The contribution of GSTs to the detoxification of carcinogens is also well documented [30]. Hence, many researchers have examined the risk of oral squamous cell carcinoma conferred by the CYP1A1, GSTM1, and GSTT1 polymorphisms, and these efforts have been summarized in 3 meta-analyses [18-20]. Although one meta-analysis concluded that CYP1A1 polymorphisms might not be risk factors for oral cancer, another meta-analysis showed significantly increased risks of oral cancer related to the CYP1A $1 * 2 \mathrm{~A}$ 
(MspI site) homozygous variant $(\mathrm{OR}=2.0)$ and the CYP1A $1 * 2 \mathrm{C} \mathrm{G} / \mathrm{G}$ genotype in people who had ever smoked $(\mathrm{OR}=2.2)$ [18]. Among these 3 studies investigating the contribution of the CYP1A $1 * 2 \mathrm{C}$ polymorphism to OPMD [26,27,31], only one reported a significant association with the CYP1A1*2C G allele $(\mathrm{G} / \mathrm{G}$ and $\mathrm{A} / \mathrm{G}$ vs $\mathrm{A} / \mathrm{A} ; \mathrm{OR}=2.67$ and 15.2, respectively) [26]. Two studies showed no significant association between the CYP1A $1 * 2 \mathrm{~A}$ polymorphism and OPMD risk [32-34]. The lack of consistency in these reports warrants further research to clarify the roles of these variants in the risk of OPMD. We found that the CYP1A1*2C A/A genotype conferred an increased OPMD risk. We also observed a further increase in risk among those who smoked more cigarettes, indicating an interaction with tobacco exposure. However, the interaction was moderately significant for OPMD $(P=0.09)$ and statistically significant for leukoplakia $(P=0.039)$. Smoking is a major risk factor for OPMD, whereas CYP1A1 plays a role in metabolizing tobacco-derived PAHs. Further research with a large sample should investigate the interaction between CYP1A $1 * 2 \mathrm{C}$ polymorphism and cigarette smoking on the risk of OPMD, and particularly the leukoplakia subgroup. We found no association between the CYP2E1*PstI/RsaI polymorphism and OPMD risk, which is in agreement with previous studies [27,31,34].

Although a meta-analysis of 19 studies reported no association between the GSTM1-null genotype and oral and pharyngeal cancers [18], 2 recent metaanalyses of more than 27 case-control studies suggested that the GSTM1-null genotype was associated with a higher risk of oral cancer in Asians but not Caucasians [19,20]. For OPMD, 5 studies reported an increased risk [27,35-38], and 5 showed no association [32-34,39,40]. In this study, the GSTM1null genotype was a risk factor for OPMD (mainly for leukoplakia), especially for heavy smokers. Our findings regarding the GSTT1 polymorphism are consistent with a meta-analysis of 19 oral cancer studies, which failed to find a significant relationship [20]. However, the genetic effect of the GSTT1-null genotype on OPMD risk was higher in 5 studies [7,15,18, $19,28]$, lower in $2[8,10]$, and unchanged in 2 studies $[14,30]$. The lack of a significant relationship between the GSTP1 genotype and OPMD in this study is consistent with 3 previous reports [27,34,39]. Discrepancies among these studies investigating the role of xenobiotic-metabolizing genes on the OPMD risk may be the result of small sample sizes or ethnic diversity.
This study shows that the polymorphisms of GSTM1 and $\mathrm{CYP} 1 \mathrm{~A} 1 * 2 \mathrm{C}$ genes and their interactions with cigarette smoking are significantly associated with an increased risk of leukoplakia, but not submucous fibrosis. Previous research has shown that tobacco use corresponds to $80 \%$ of cases of oral leukoplakia, whereas betel nut chewing is the main contributor to oral submucous fibrosis $[6,41]$. We observed a distinct genetic predisposition between leukoplakia and submucous fibrosis, suggesting that risk factors differ for these 2 subtypes of OPMD. Further studies on gene-betel nut interactions would be helpful in clarifying the etiological factors of oral submucous fibrosis.

This study reveals the combined effects of multiple polymorphisms of xenobiotic-metabolizing genes regarding OPMD. With the complexity of environmental exposure to various carcinogens, the effective biotransformation of chemical mixtures may require multiple metabolizing genes. Activation overexpression or detoxification failure of metabolizing enzymes for chemical carcinogens may increase cancer risk. Although no study has examined the risk conferred by the combination of multiple metabolizing genes, 3 studies on gene-gene interactions have shown that the simultaneous presence of both GSTM1 and GSTT1 deletions is associated with an increased risk of developing oral leukoplakia $[27,35,38]$. These findings demonstrate that the simultaneous presence of more unfavorable genotypes of xenobiotic-metabolizing genes may increase susceptibility to OPMD.

This study has a few limitations. First, the majority of prisoners tested had heavy smoking histories and were prone to betel nut and alcohol use. These environmental factors may have confounded the association between genetic polymorphisms and OPMD risk. The multivariate logistic regression models in this study included these 3 factors to estimate adjusted ORs for the risk of OPMD associated with genetic polymorphisms. If these environmental factors confounded the true genetic effects of OPMD risk, the enrollment of participants with a high percentage of these habits may have led the estimated ORs toward the null, further strengthening our findings. Therefore, we are confident of the genetic predisposition to oral lesions. Second, factors such as nutrition and psychological status also play important roles in the etiology of oral lesions $[42,43]$, although these were not evaluated in our study. Therefore, we cannot rule out the potentially confounding effects of these factors. However, no convincing evidences to support that these variables might be related to the polymorphisms of xenobiotic-metabolizing 
genes or the risk of oral lesions. Hence, these factors may not confound our results. Third, all participants were male prisoners of Taiwanese ethnicity. Therefore, the ability to generalize these results to the general Taiwanese population and other racial/ethnic groups is unclear. Further studies on the general population of Taiwan or another ethnic population are needed to confirm the findings. Fourth, leukoplakia is a fairly general term in oral pathology, and it represents a wide range of pathologic conditions that are mostly not premalignant. Thus, it may not be possible to draw any real conclusions regarding the genetic nature of leukoplakia. Finally, recall bias is another source of information bias in this study. However, the recall bias may have been similar between cases and controls under the standardized study procedure adopted in this study.

The results of this study suggest that genetic polymorphisms of the GSTM 1 and CYP1A $1 * 2 \mathrm{C}$ genes, particularly combined with more than 3 xenobioticmetabolizing genes, may be associated with susceptibility to leukoplakia. However, further studies incorporating a larger sample size and another ethnic population are needed to confirm this association.

\section{Acknowledgments}

The authors are grateful to the study participants. This study was supported by grants CMU95-324, CMU97-119, and CMU97-121 from China Medical University, NSC97-2314-B-039-021-MY3 from the National Science Council, and DOH 100-TD-B-111004, DOH100-TD-C-111-005, and DOH101-TD-C111-008 from the Department of Health, Taiwan.

\section{References}

[1] A. Jemal, F. Bray, M.M. Center, J. Ferlay, E. Ward and D. Forman, Global cancer statistics, CA Cancer J Clin 61 (2011), 69-90.

[2] Department of Health, Health and Vital Statistics, Department of Health, Executive Yuan, Taipei, 2011.

[3] S.S. Hsue, W.C. Wang, C.H. Chen, C.C. Lin, Y.K. Chen and L.M. Lin, Malignant transformation in 1458 patients with potentially malignant oral mucosal disorders: A follow-up study based in a Taiwanese hospital, J Oral Pathol Med 36 (2007), 25-29.

[4] M.N. Shiu and T.H. Chen, Intervention efficacy and malignant transformation to oral cancer among patients with leukoplakia (Review), Oncol Rep 10 (2003), 1683-1692.

[5] P.O. Lind, Malignant transformation in oral leukoplakia, Scand J Dent Res 95 (1987), 449-455.

[6] B.W. Neville and T.A. Day, Oral cancer and precancerous lesions, CA Cancer J Clin 52 (2002), 195-215.
[7] C.H. Chung, Y.H. Yang, T.Y. Wang, T.Y. Shieh and S. Warnakulasuriya, Oral precancerous disorders associated with areca quid chewing, smoking, and alcohol drinking in southern Taiwan, J Oral Pathol Med 34 (2005), 460-466.

[8] A. Ariyawardana, A.D. Athukorala and A. Arulanandam, Effect of betel chewing, tobacco smoking and alcohol consumption on oral submucous fibrosis: A case-control study in Sri Lanka, J Oral Pathol Med 35 (2006), 197-201.

[9] J.J. Lee, H.C. Hung, S.J. Cheng, Y.J. Chen, C.P. Chiang, B.Y. Liu et al., Carcinoma and dysplasia in oral leukoplakias in Taiwan: prevalence and risk factors, Oral Surg Oral Med Oral Pathol Oral Radiol Endod 101 (2006), 472-480.

[10] R. Muwonge, K. Ramadas, R. Sankila, S. Thara, G. Thomas, J. Vinoda et al., Role of tobacco smoking, chewing and alcohol drinking in the risk of oral cancer in Trivandrum, India: A nested case-control design using incident cancer cases, Oral Oncol 44 (2008), 446-454.

[11] Y.C. Ko, Y.L. Huang, C.H. Lee, M.J. Chen, L.M. Lin and C.C. Tsai, Betel quid chewing, cigarette smoking and alcohol consumption related to oral cancer in Taiwan, J Oral Pathol Med 24 (1995), 450-453.

[12] H. Bartsch and E. Hietanen, The role of individual susceptibility in cancer burden related to environmental exposure, Environ Health Perspect 104(Suppl 3) (1996), 569-577.

[13] H. Raunio, K. Husgafvel-Pursiainen, S. Anttila, E. Hietanen, A. Hirvonen and O. Pelkonen, Diagnosis of polymorphisms in carcinogen-activating and inactivating enzymes and cancer susceptibility - a review, Gene 159 (1995), 113-121.

[14] H. Bartsch, U. Nair, A. Risch, M. Rojas, H. Wikman and K. Alexandrov, Genetic polymorphism of CYP genes, alone or in combination, as a risk modifier of tobacco-related cancers, Cancer Epidemiol Biomarkers Prev 9 (2000), 3-28.

[15] T.J. Smith, A. Liao, L.D. Wang, G.Y. Yang, S. Starcic, M.A. Philbert et al., Characterization of xenobiotic-metabolizing enzymes and nitrosamine metabolism in the human esophagus, Carcinogenesis 19 (1998), 667-672.

[16] T.Y. Liu, Y.T. Chung, P.F. Wang, C.W. Chi and L.L. Hsieh, Safrole-DNA adducts in human peripheral blood - an association with areca quid chewing and CYP2E1 polymorphisms, Mutat Res 559 (2004), 59-66.

[17] M. Deakin, J. Elder, C. Hendrickse, D. Peckham, D. Baldwin, C. Pantin et al., Glutathione S-transferase GSTT1 genotypes and susceptibility to cancer: Studies of interactions with GSTM1 in lung, oral, gastric and colorectal cancers, Carcinogenesis 17 (1996), 881-884.

[18] L. Varela-Lema, E. Taioli, A. Ruano-Ravina, J.M. BarrosDios, D. Anantharaman, S. Benhamou et al., Meta-analysis and pooled analysis of GSTM1 and CYP1A1 polymorphisms and oral and pharyngeal cancers: A HuGE-GSEC review, Genet Med 10 (2008), 369-384.

[19] W. Zhuo, Y. Wang, X. Zhuo, Y. Zhu, W. Wang, B. Zhu et al., CYP1A1 and GSTM1 polymorphisms and oral cancer risk: Association studies via evidence-based meta-analyses, Cancer Invest 27 (2009), 86-95.

[20] Z.J. Zhang, K. Hao, R. Shi, G. Zhao, G.X. Jiang, Y. Song et al., Glutathione S-transferase M1 (GSTM1) and glutathione S-transferase T1 (GSTT1) null polymorphisms, smoking, and their interaction in oral cancer: A HuGE review and metaanalysis, Am J Epidemiol 173 (2011), 847-857.

[21] S. Warnakulasuriya, N.W. Johnson and I. van der Waal, Nomenclature and classification of potentially malignant disorders of the oral mucosa, J Oral Pathol Med 36 (2007), 575580 .

[22] C.C. Yeh, L.L. Hsieh, R. Tang, C.R. Chang-Chieh and 
F.C. Sung, Vegetable/fruit, smoking, glutathione S-transferase polymorphisms and risk for colorectal cancer in Taiwan, World J Gastroenterol 11 (2005), 1473-1480.

[23] C.C. Yeh, F.C. Sung, L.T. Kuo, W.P. Hsu and H.Y. Chu, Polymorphisms of cytochrome P450 1A1, cigarette smoking and risk of coronary artery disease, Mutat Res 667 (2009), 77-81.

[24] G.J. Gattas, M.B. de Carvalho, M.S. Siraque, O.A. Curioni, P. Kohler, J. Eluf-Neto et al., Genetic polymorphisms of CYP1A1, CYP2E1, GSTM1, and GSTT1 associated with head and neck cancer, Head Neck 28 (2006), 819-826.

[25] C.C. Yeh, F.C. Sung, R. Tang, C.R. Chang-Chieh and L.L. Hsieh, Association between polymorphisms of biotransformation and DNA-repair genes and risk of colorectal cancer in Taiwan, J Biomed Sci 14 (2007), 183-193.

[26] S.Y. Kao, C.H. Wu, S.C. Lin, S.K. Yap, C.S. Chang, Y.K. Wong et al., Genetic polymorphism of cytochrome P4501A1 and susceptibility to oral squamous cell carcinoma and oral precancer lesions associated with smoking/betel use, J Oral Pathol Med 31 (2002), 505-511.

[27] E.C. Duarte, D.C. Ribeiro, M.V. Gomez, M.L. Ramos-Jorge and R.S. Gomez, Genetic polymorphisms of carcinogen metabolizing enzymes are associated with oral leukoplakia development and p53 overexpression, Anticancer Res 28 (2008), 1101-1106.

[28] F.P. Guengerich, Roles of cytochrome P-450 enzymes in chemical carcinogenesis and cancer chemotherapy, Cancer Res 48 (1988), 2946-2954.

[29] M. Vondracek, Z. Xi, P. Larsson, V. Baker, K. Mace, A. Pfeifer et al., Cytochrome P450 expression and related metabolism in human buccal mucosa, Carcinogenesis 22 (2001), 481-488.

[30] B. Ketterer, Protective role of glutathione and glutathione transferases in mutagenesis and carcinogenesis, Mutat Res 202 (1988), 343-361.

[31] N. Sikdar, S.A. Mahmud, R.R. Paul and B. Roy, Polymorphism in CYP1A1 and CYP2E1 genes and susceptibility to leukoplakia in Indian tobacco users, Cancer Lett 195 (2003), 33-42.

[32] D. Anantharaman, P.M. Chaubal, S. Kannan, R.A. Bhisey and M.B. Mahimkar, Susceptibility to oral cancer by genetic polymorphisms at CYP1A1, GSTM1 and GSTT1 loci among Indians: tobacco exposure as a risk modulator, Carcinogenesis 28 (2007), 1455-1462.
[33] S. Chatterjee, S. Chakrabarti, B. Sengupta, S. Poddar, D. Biswas, S. Sengupta et al., Prevalence of CYP1A1 and GST polymorphisms in the population of northeastern India and susceptibility of oral cancer, Oncol Res 17 (2009), 397-403.

[34] D. Anantharaman, T.A. Samant, S. Sen and M.B. Mahimkar, Polymorphisms in tobacco metabolism and DNA repair genes modulate oral precancer and cancer risk, Oral Oncol 47 (2011), 866-872.

[35] U.J. Nair, J. Nair, B. Mathew and H. Bartsch, Glutathione Stransferase M1 and T1 null genotypes as risk factors for oral leukoplakia in ethnic Indian betel quid/tobacco chewers, $\mathrm{Car}$ cinogenesis 20 (1999), 743-748.

[36] E.C. Duarte, M.S. da Silva, M.V. Gomez and R.S. Gomez, GSTM1 polymorphism and oral leukoplakia, J Oral Pathol Med 35 (2006), 202-205.

[37] B.K. Yadav, J. Kaur, A. Srivastava and R. Ralhan, Effect of polymorphisms in XRCC1, CCND1 and GSTM1 and tobacco exposure as risk modifier for oral leukoplakia, Int J Biol Markers 24 (2009), 90-98.

[38] D. Agrawal, S. Gupta, D. Agarwal, O.P. Gupta and M. Agarwal, Role of GSTM1 and GSTT1 polymorphism: susceptibility to oral submucous fibrosis in the North Indian population, Oncology 79 (2010), 181-186.

[39] N. Sikdar, R.R. Paul and B. Roy, Glutathione S-transferase M3 (A/A) genotype as a risk factor for oral cancer and leukoplakia among Indian tobacco smokers, Int J Cancer 109 (2004), 95-101.

[40] R.J. Bathi, R. Rao and S. Mutalik, GST null genotype and antioxidants: Risk indicators for oral pre-cancer and cancer, Indian J Dent Res 20 (2009), 298-303.

[41] P.R. Murti, R.B. Bhonsle, P.C. Gupta, D.K. Daftary, J.J. Pindborg and F.S. Mehta, Etiology of oral submucous fibrosis with special reference to the role of areca nut chewing, $J$ Oral Pathol Med 24 (1995), 145-152.

[42] M. Soto Araya, G. Rojas Alcayaga and A. Esguep, Association between psychological disorders and the presence of Oral lichen planus, Burning mouth syndrome and Recurrent aphthous stomatitis, Med Oral 9 (2004), 1-7.

[43] P.A. Reichart, Identification of risk groups for oral precancer and cancer and preventive measures, Clin Oral Investig 5 (2001), 207-213. 


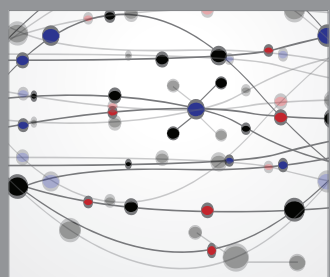

The Scientific World Journal
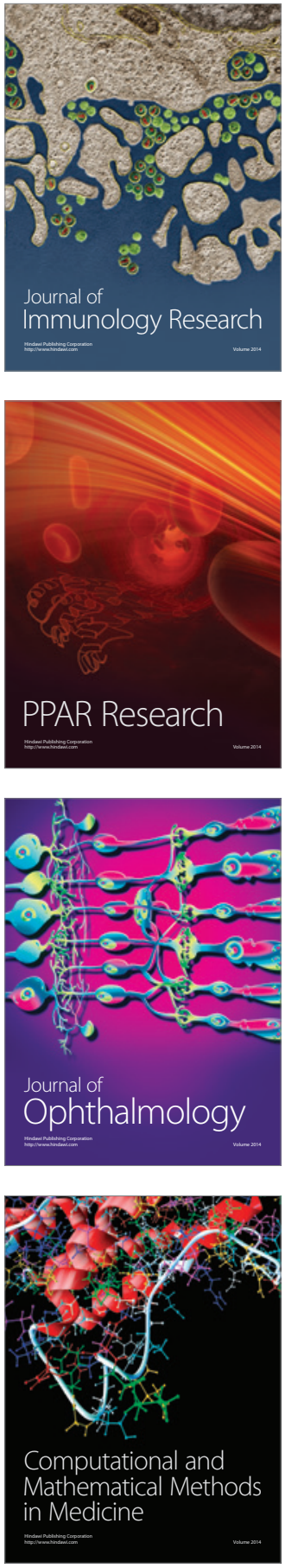

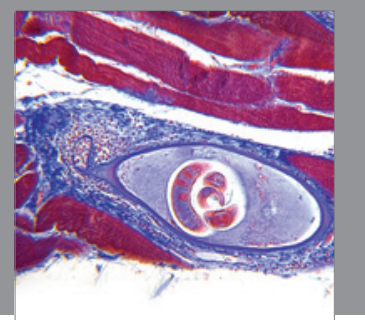

Gastroenterology

Research and Practice
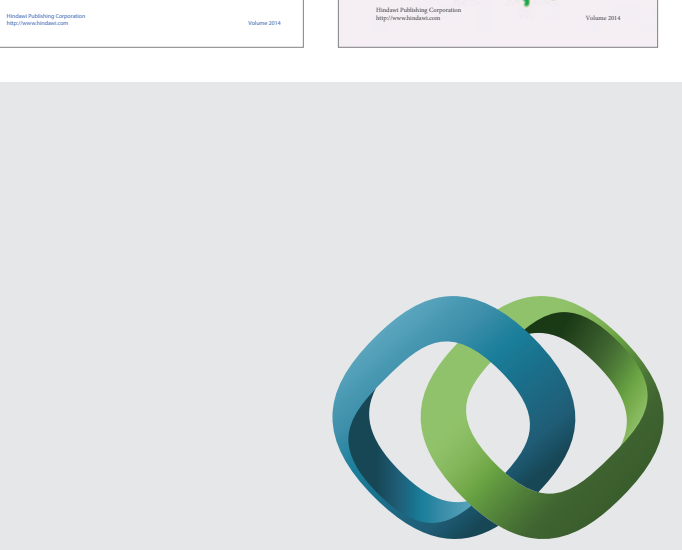

\section{Hindawi}

Submit your manuscripts at

http://www.hindawi.com
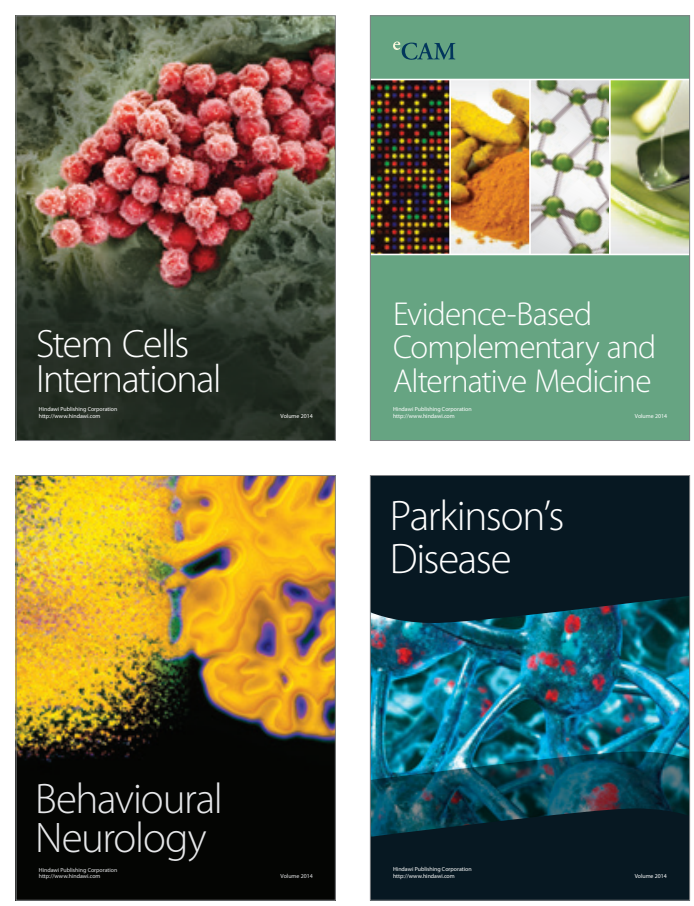

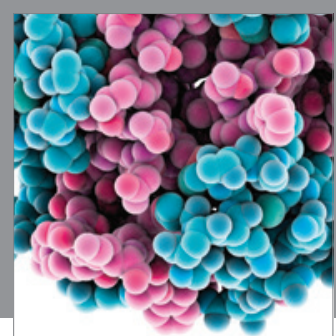

Journal of
Diabetes Research

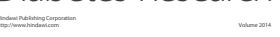

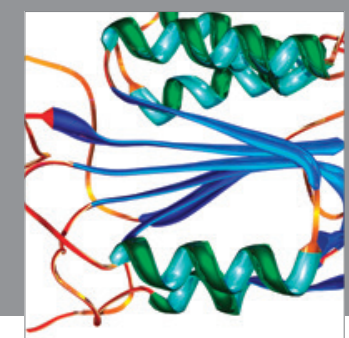

Disease Markers
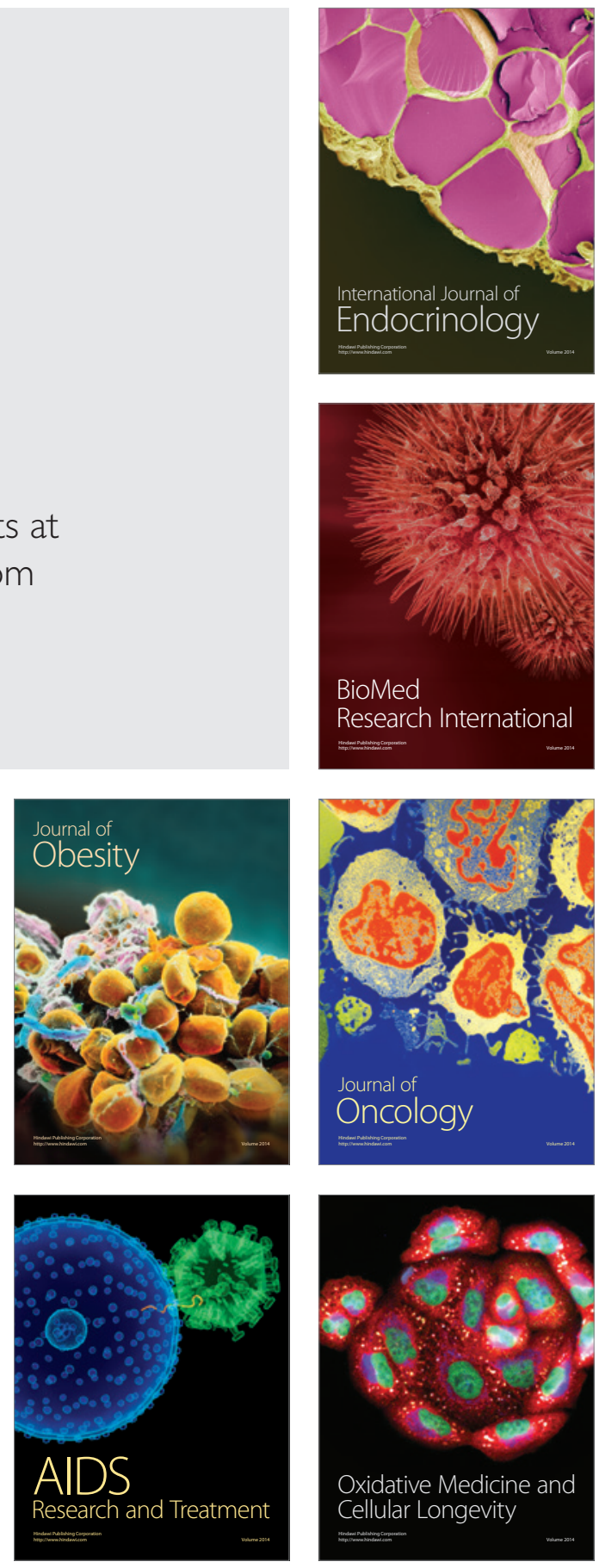